\title{
Monte Carlo simulations of dusty gas discs around supermassive black holes
}

\author{
Maarten Baes ${ }^{1}$ and Sławomir Piasecki ${ }^{1,2}$ \\ ${ }^{1}$ Universiteit Gent, Belgium, email: maarten.baes@ugent.be \\ ${ }^{2}$ Adam Mickiewicz University, Poznań, Poland
}

\begin{abstract}
Using detailed Monte Carlo radiative transfer simulations in realistic models for galactic nuclei, we critically investigate the influence of interstellar dust in ionised gas discs on the rotation curves and the resulting black hole mass measurements. We find that absorption and scattering by interstellar dust leaves the shape of the rotation curves basically unaltered, but slightly decreases the central slope of the rotation curves. As a result, the "observed" black hole masses are systematically underestimated by some 10 to $20 \%$ for realistic optical depths.
\end{abstract}

Keywords. Galaxies: nuclei - black hole physics

\section{Introduction}

Measuring the kinematics of ionised gas disks has become one the most important methods to determine the masses of supermassive black holes (SMBHs) in the nuclei of nearby galaxies. The state-of-the-art modeling techniques are refined to a high degree and take into account the major relevant astrophysical processes as well as the main instrumental effects. Applying such modeling techniques to high-quality HST data, a formal SMBH mass measurement uncertainty of $25 \%$ or better has been claimed with well-behaved disks (e.g. Barth et al. 2001; Marconi et al. 2003; Atkinson et al. 2005; Häring-Neumayer et al. 2006). Many ionised gas disks contain substantial amounts of interstellar dust. As optical radiation is easily absorbed and scattered by interstellar dust grains, these effects might affect the observed rotation curve and hence the SMBH mass estimate. The goal of our investigation is to determine the importance of this effect through detailed Monte Carlo simulations.

\section{The modelling approach}

We have set up a detailed radiative transfer model to investigate the effects of neglecting dust absorption and scattering on ionised gas disc rotation curves and the corresponding SMBH masses in a typical galactic nucleus. Our model consists of a thin (but not infinitesimally thin) axisymmetrical double-exponential disc rotating in a galactic nucleus. The intrinsic rotation velocity of the disc is determined by the combined gravitational potential of a stellar distribution and a central SMBH. We consider various $\mathrm{SMBH}$ masses. An additional isotropic bulk velocity dispersion is taken into account. We assume that a fraction of the disc consists of interstellar dust with typical Milky Way dust optical properties. The dust is assumed to be coupled to the ionised gas and has exactly the same spatial and velocity distribution. The SMBH mass $M_{\bullet}$, the thickness $z_{0}$ of the disc and the $\mathrm{V}$-band optical depth $\tau_{\mathrm{v}}$, a quantity equivalent to the dust mass, are free parameters in our models.

For each model, we calculate the observed line-of-sight velocity field (data cubes) at several inclination angles. We use the SKIRT code, a 3D radiative transfer code that has 

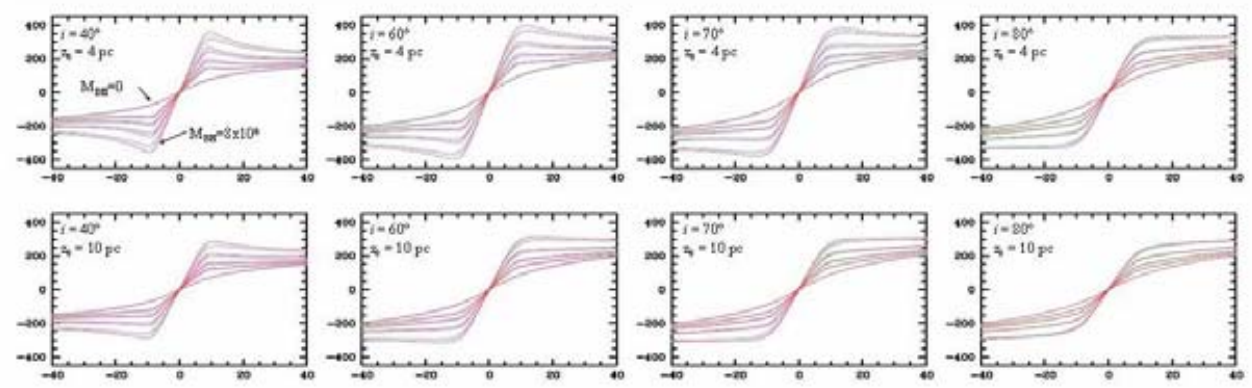

Figure 1. Nuclear rotation curves for dusty ionised gas discs. Each panel corresponds to a particular choice for the inclination $i$ and the disc scale height $z_{0}$. In each panel we plot the rotation curves for different black hole masses (ranging between 0 and $8 \times 10^{8} \mathrm{M}_{\odot}$ ) and for different optical depths (ranging between 0 and 2). For any $i, z_{0}$ and $M_{\bullet}$, the slope of the rotation curve slightly decreases with increasing optical depth.

been developed explicitly to model the kinematics of dusty galaxies (Baes \& Dejonghe 2002; Baes et al. 2003). The effects of absorption and scattering are properly taken into account. From the data cubes, we calculate rotation velocity maps and velocity dispersion maps. We focus in particular on the major axis rotation curves.

\section{Results and conclusions}

In Figure 1 we plot a set of nuclear rotation curves for our dusty ionised gas discs. The different panels correspond to different values for $z_{0}$ and the inclination angle. Within each individual panel, rotation curves are shown for different values of $M_{\bullet}$ and $\tau_{\mathrm{v}}$. For a fixed spatial resolution and inclination, the shape of the rotation curve of dust-free models depends on the SMBH mass and disc thickness. In particular, increasing disc thickness has a significant influence due to the larger integration along the line of sight. Modeling ionised gas disks as infinitesimally thin disks might introduce a bias.

To investigate the effect of interstellar dust absorption and scattering, we must compare models with different values of the optical depth. The curves corresponding to various $\tau_{\mathrm{V}}$ basically coincide: apparently absorption and scattering by interstellar dust leaves the shape of the rotation curves qualitatively unaltered. However, close inspection shows that an increasing amount of interstellar dust slightly decreases the central slope of the rotation curves. We estimate the bias one makes by neglecting the effect of interstellar dust on SMBH mass estimates by translating the observed rotation curve central slopes into SMBH masses. Neglecting dust systematically leads to an underestimate of the black hole mass. For $\tau_{\mathrm{V}} \sim 0.2$ the SMBH is underestimated by about $10 \%$, for $\tau_{\mathrm{V}}>1$ the black hole mass is underestimated by 10 to $20 \%$. This effect is more or less independent of the SMBH mass and the inclination of the disc. Our study demonstrates that the systematic effect of dust attenuation should be taken into account in SMBH demographics studies.

\section{References}

Atkinson, J. W., Collett, J. L., Marconi, A. et al. 2005, MNRAS, 359, 504

Baes, M. \& Dejonghe, H. 2002, MNRAS, 335, 441

Baes, M., Davies, J. I., Dejonghe, H. et al. 2003, MNRAS, 343, 1081

Barth, A. J., Sarzi, M., Rix, H.-W. et al. 2001, ApJ, 555, 685

Häring-Neumayer, N., Cappellari, M., Rix, H.-W. et al. 2006, ApJ, 643, 226

Marconi, A., Axon, D. J., Capetti, A. et al. 2003, ApJ, 586, 868 